\title{
Utilization of an Oxonia-Cope Rearrangement as a Mechanistic Probe for Prins Cyclizations
}

\author{
Ramesh Jasti, Christopher D. Anderson, and Scott D. Rychnovsky* \\ Department of Chemistry, University of California, Irvine, Irvine, CA 92697-2025
}

\section{Supporting Information}

\begin{tabular}{ll} 
Table of Contents & Pages \\
\hline Complete Reference 20 & S2 \\
General Experimental & S2-S3 \\
Preparation of Esters & S3-S5 \\
Preparation of Acetoxy Ethers & S5-S8 \\
Prins Cyclizations & S9-S16 \\
Analysis of Enantiomeric Excess & S16-S17 \\
Optimized Geometries & S17-S26
\end{tabular}




\section{Complete Reference 20.}

Frisch, M. J.; Trucks, G. W.; Schlegel, H. B.; Scuseria, G. E.; Robb, M. A.; Cheeseman, J. R.; Montgomery, Jr., J. A.; Vreven, T.; Kudin, K. N.; Burant, J. C.; Millam, J. M.; Iyengar, S. S.; Tomasi, J.; Barone, V.; Mennucci, B.; Cossi, M.; Scalmani, G.; Rega, N.; Petersson, G. A.; Nakatsuji, H.; Hada, M.; Ehara, M.; Toyota, K.; Fukuda, R.; Hasegawa, J.; Ishida, M.; Nakajima, T.; Honda, Y.; Kitao, O.; Nakai, H.; Klene, M.; Li, X.; Knox, J. E.; Hratchian, H. P.; Cross, J. B.; Bakken, V.; Adamo, C.; Jaramillo, J.; Gomperts, R.; Stratmann, R. E.; Yazyev, O.; Austin, A. J.; Cammi, R.; Pomelli, C.; Ochterski, J. W.; Ayala, P. Y.; Morokuma, K.; Voth, G. A.; Salvador, P.; Dannenberg, J. J.; Zakrzewski, V. G.; Dapprich, S.; Daniels, A. D.; Strain, M. C.; Farkas, O.; Malick, D. K.; Rabuck, A. D.; Raghavachari, K.; Foresman, J. B.; Ortiz, J. V.; Cui, Q.; Baboul, A. G.; Clifford, S.; Cioslowski, J.; Stefanov, B. B.; Liu, G.; Liashenko, A.; Piskorz, P.; Komaromi, I.; Martin, R. L.; Fox, D. J.; Keith, T.; Al-Laham, M. A.; Peng, C. Y.; Nanayakkara, A.; Challacombe, M.; Gill, P. M. W.; Johnson, B.; Chen, W.; Wong, M. W.; Gonzalez, C.; and Pople, J. A. Gaussian 03, Revision C.02; Gaussian, Inc., Wallingford CT, 2004.

General Experimental Details. ${ }^{1} \mathrm{H}$ NMR spectra were recorded at 400 or $500 \mathrm{MHz}$ on Bruker NMR spectrometers and were referenced to residual solvent peaks. ${ }^{13} \mathrm{C}$ NMR spectra were recorded at $125 \mathrm{MHz}$ on Bruker NMR spectrometers. Infrared spectra were recorded on a MIDAC Grams/Prospect FT-IR. Optical rotations were measured on a JASCO DIP-370 digital polarimiter. Enantiomeric excess was assessed by Hewlett Packard Series 1050 HPLC with Chiracel OD-H column utilizing a hexane/isopropanol mobile phase. All reagents were obtained commercially and purified prior to use. Tetrahydrofuran, diethyl ether, dichloromethane, and toluene were dried by filtration through alumina according to the methods described by Grubbs. ${ }^{1}$ Acetic anhydride was refluxed over powdered potassium carbonate for $2 \mathrm{hr}$ then distilled at atmospheric pressure. Liquid chromatography was performed using forced flow of the indicated solvent system on reagent silica gel 60. Thin layer chromatography (TLC) was performed on Whatman $250 \mu \mathrm{m}$ layer silica gel plates. Developed plates were visualized using $\rho$-anisaldehyde, potassium permanganate, $I_{2}$ stains and UV light. All glassware

\footnotetext{
${ }^{1}$ Pangborn, A.B.; Giardello, M. A.; Grubbs, R. H.; Rosen, R. K.; Timmers, F. J. Organometallics 1996, $15,1518-1520$.
} 
was flame-dried and cooled under an inert atmosphere of argon unless otherwise stated. Moisture sensitive reactions were carried out under an inert atmosphere of argon using standard syringe/septa techniques.

\section{Procedures and Characterization}

\section{Representative Procedure for Esterification of Homoallylic Alcohols:}

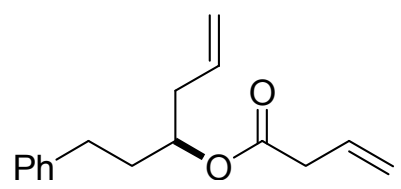

24

Ester 24. Alcohol 23 (4.13 g, $23.5 \mathrm{mmol}, 1$ equiv) was dissolved in $250 \mathrm{~mL}$ of $\mathrm{CH}_{2} \mathrm{Cl}_{2}$. To this solution was added 3-propenoic acid (2.63 g, $30.5 \mathrm{mmol}, 1.3$ equiv) and DMAP ( $0.87 \mathrm{~g}, 7.1 \mathrm{mmol}, 0.3$ equiv). The reaction mixture was then cooled to $0{ }^{\circ} \mathrm{C}$ whereupon DCC (5.82 g, $28.2 \mathrm{mmol}, 1.2$ equiv) was added. The mixture was stirred vigorously overnight and filtered. The eluent was concentrated and chromatographed (1-10\% EtOAc/Hexanes) to provide $5.32 \mathrm{~g}(93 \%)$ of ester 24: $[\alpha]^{24}{ }_{\mathrm{D}}-13.6\left(c 1.0, \mathrm{CHCl}_{3}\right)$; IR (neat) $3081,3027,2931,1736,1643,1496,1173 \mathrm{~cm}^{-1} ;{ }^{1} \mathrm{H}$ NMR $\left(500 \mathrm{MHz}, \mathrm{CDCl}_{3}\right) \delta$ 7.23 (m, 5 H), 5.94 (m, $1 \mathrm{H}), 5.76$ (dddd, $J=17.2,10.2,7.1,7.0 \mathrm{~Hz}, 1 \mathrm{H}), 5.20$ (dddd, $J=$ 7.1, 2.6, 1.3, $0.2 \mathrm{~Hz}, 1 \mathrm{H}), 5.17$ (t, $J=1.5 \mathrm{~Hz}, 1 \mathrm{H}), 5.10(\mathrm{~m}, 1 \mathrm{H}), 5.07$ (m, $1 \mathrm{H}), 5.00$ (m, $1 \mathrm{H}), 3.08$ (ddd, $J=7.0,1.4,1.4 \mathrm{~Hz}, 2 \mathrm{H}), 2.68$ (ddd, $J=13.8,9.8,6.1,1 \mathrm{H}), 2.61$ (ddd, $J=13.8,9.5,6.8,1 \mathrm{H}), 2.37(\mathrm{~m}, 2 \mathrm{H}), 1.90(\mathrm{~m}, 2 \mathrm{H}) ;{ }^{13} \mathrm{C}$ NMR $\left(125 \mathrm{MHz}, \mathrm{CDCl}_{3}\right)$ $\delta 171.1,141.4,133.3,130.4,128.4,128.3,125.9,118.4,117.9,73.0,39.3,38.6,35.2$, 31.7; HRMS (CI/NH$\left.)_{3}\right) m / z$ calcd for $\mathrm{C}_{13} \mathrm{H}_{15} \mathrm{O}_{2}\left(\mathrm{M}-\mathrm{C}_{3} \mathrm{H}_{5}\right)^{+}$203.1072, found 203.1072. Anal. Calcd for $\mathrm{C}_{16} \mathrm{H}_{20} \mathrm{O}_{2}$ : C, 78.65; H, 8.25. Found: C, 78.47; H, 7.98. 


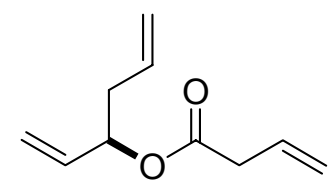

55

Ester 55. $[\alpha]^{24} 8.8\left(c 5.0, \mathrm{CHCl}_{3}\right)$; IR (neat) $3083,1739,1643,1426,1172 \mathrm{~cm}^{-1} ;{ }^{1} \mathrm{H}$ NMR (500 MHz, CDCl $) \delta 5.93(\mathrm{~m}, 1 \mathrm{H}), 5.77(\mathrm{~m}, 2 \mathrm{H}), 5.33(\mathrm{q}, J=6.3 \mathrm{~Hz}, 1 \mathrm{H}), 5.26$ (dt, $J=17.3,1.3 \mathrm{~Hz}, 1 \mathrm{H}), 5.15$ (m, $5 \mathrm{H}), 3.11$ (dt, $J=7.0,1.4 \mathrm{~Hz}, 2 \mathrm{H}), 2.37$ (t, $J=5.6$ $\mathrm{Hz}, 2 \mathrm{H}) ;{ }^{13} \mathrm{C}$ NMR $\left(125 \mathrm{MHz}, \mathrm{CDCl}_{3}\right) \delta 170.6,135.8,133.0,130.2,118.5,118.0,116.9$, 73.9, 39.3, 38.7; LRMS (ESI) $m / z$ calcd for $\mathrm{C}_{10} \mathrm{H}_{15} \mathrm{O}_{2}(\mathrm{M}+\mathrm{H})^{+} 167$, found 167 .

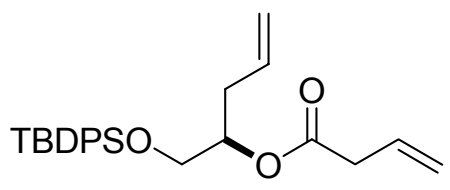

56

Ester 56. $[\alpha]^{24}{ }_{\mathrm{D}}-6.9\left(c\right.$ 4.4, $\left.\mathrm{CHCl}_{3}\right)$; IR (neat) $2958,1739,1428,1113 \mathrm{~cm}^{-1} ;{ }^{1} \mathrm{H} \mathrm{NMR}$ (500 MHz, $\left.\mathrm{CDCl}_{3}\right) \delta 7.67(\mathrm{~m}, 4 \mathrm{H}), 7.42(\mathrm{~m}, 6 \mathrm{H}), 5.92(\mathrm{ddt}, J=16.9,9.8,7.0 \mathrm{~Hz}, 1 \mathrm{H})$, $5.73(\mathrm{ddt}, J=17.2,10.1,7.1 \mathrm{~Hz}, 1 \mathrm{H}), 5.12(\mathrm{~m}, 5 \mathrm{H}), 3.72(\mathrm{~m}, 2 \mathrm{H}), 3.07$ (m, $2 \mathrm{H}), 2.47$ (dt, $J=14.1,6.2 \mathrm{~Hz}, 1 \mathrm{H}), 2.37$ (dt, $J=14.3,7.2 \mathrm{~Hz}, 1 \mathrm{H}), 1.06(\mathrm{~s}, 9 \mathrm{H}) ;{ }^{13} \mathrm{C}$ NMR $(125$ $\left.\mathrm{MHz}, \mathrm{CDCl}_{3}\right) \delta 171.0,135.6,135.5,133.4,133.3,130.4,129.7$ (2), 127.8, 127.7, 127.6, 118.4, 117.9, 73.7, 64.4, 39.3, 35.0, 26.7, 19.2; HRMS (ESI) $m / z$ calcd for $\mathrm{C}_{25} \mathrm{H}_{32} \mathrm{O}_{3} \mathrm{Si}$ $(\mathrm{M}+\mathrm{Na})^{+} 431.2018$, found 431.2025 .

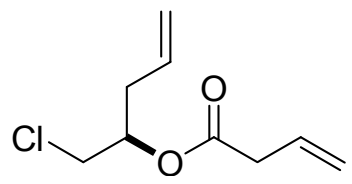


Ester 57. $[\alpha]^{24} 7.5\left(c\right.$ 14.5, $\mathrm{CHCl}_{3}$ ); IR (neat) 282, 2360, 1742, 1643, $1168 \mathrm{~cm}^{-1} ;{ }^{1} \mathrm{H}$ NMR $\left(500 \mathrm{MHz}, \mathrm{CDCl}_{3}\right) \delta 5.92(\mathrm{ddt}, J=17.5,9.8,6.9 \mathrm{~Hz}, 1 \mathrm{H}), 5.72(\mathrm{ddt}, J=17.2$, 10.2, 7.1 Hz, $1 \mathrm{H}), 5.15(\mathrm{~m}, 5 \mathrm{H}), 3.64$ (dd, $J=11.7,4.6 \mathrm{~Hz}, 1 \mathrm{H}), 3.58(\mathrm{dd}, J=11.7,5.5$ $\mathrm{Hz}, 1 \mathrm{H}), 3.14$ (q, $J=1.4 \mathrm{~Hz}, 1 \mathrm{H}), 3.11$ (q, $J=1.4 \mathrm{~Hz}, 1 \mathrm{H}), 2.46(\mathrm{~m}, 2 \mathrm{H}) ;{ }^{13} \mathrm{C}$ NMR $\left(125 \mathrm{MHz}, \mathrm{CDCl}_{3}\right) \delta 170.8,132.1,129.9,119.0,118.8,72.2,44.9,39.0,35.8$; LRMS (ESI) $m / z$ calcd for $\mathrm{C}_{9} \mathrm{H}_{13} \mathrm{ClO}_{2} \mathrm{Na}(\mathrm{M}+\mathrm{Na})^{+} 211$, found 211 .

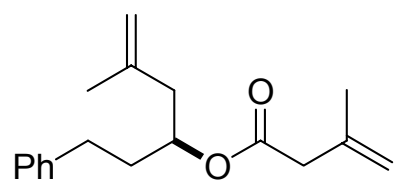

58

Ester 58. $[\alpha]^{24}{ }_{D}-6.5\left(c 7.5, \mathrm{CHCl}_{3}\right)$; IR (neat) $2933,1732,1650,1455,1153 \mathrm{~cm}^{-1} ;{ }^{1} \mathrm{H}$ NMR (500 MHz, $\left.\mathrm{CDCl}_{3}\right) \delta 7.28(\mathrm{~m}, 2 \mathrm{H}), 7.19(\mathrm{~m}, 3 \mathrm{H}), 5.13(\mathrm{dt}, J=12.6,5.9 \mathrm{~Hz}, 1 \mathrm{H})$, $4.93(\mathrm{t}, J=0.8 \mathrm{~Hz}, 1 \mathrm{H}), 4.88(\mathrm{q}, J=0.8 \mathrm{~Hz}, 1 \mathrm{H}), 4.80$ (t, $J=0.9 \mathrm{~Hz}, 1 \mathrm{H}), 4.74$ (q, $J=$ $1.0 \mathrm{~Hz}, 1 \mathrm{H}), 3.02(\mathrm{~s}, 2 \mathrm{H}), 2.70(\mathrm{dt}, J=13.8,8.0 \mathrm{~Hz}, 1 \mathrm{H}), 2.62(\mathrm{dt}, J=13.8,7.9 \mathrm{~Hz}, 1$ H), $2.36(\mathrm{dd}, J=13.9,7.5 \mathrm{~Hz}, 1 \mathrm{H}), 2.25(\mathrm{dd}, J=13.8,5.7 \mathrm{~Hz}, 1 \mathrm{H}), 1.90(\operatorname{td}, J=8.2,6.3$ $\mathrm{Hz}, 2 \mathrm{H}), 1.83$ (s, $3 \mathrm{H}), 1.74$ (s, $3 \mathrm{H}) ;{ }^{13} \mathrm{C}$ NMR $\left(125 \mathrm{MHz}, \mathrm{CDCl}_{3}\right) \delta$ 171.0, 141.6, 141.5, 138.6, 128.4, 128.3, 125.9, 114.7, 113.5, 72.0, 43.7, 42.9, 35.7, 31.8, 22.5, 22.4; HRMS (ESI) $m / z$ calcd for $\mathrm{C}_{18} \mathrm{H}_{24} \mathrm{O}_{2}(\mathrm{M}+\mathrm{Na})^{+} 295.1674$, found 295.1676 .

\section{Representative Procedure for Reductive Acetylation of Esters: ${ }^{2}$}

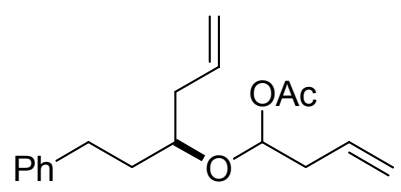

25

\footnotetext{
${ }^{2}$ Kopecky, D. J.; Rychnovsky, S. D. J. Org. Chem. 2000, 65, 191-198.
} 
Acetoxy Ether 25. Ester 24 (1.00 g, $4.10 \mathrm{mmol}, 1$ equiv) was dissolved in $40 \mathrm{~mL}$ of $\mathrm{CH}_{2} \mathrm{Cl}_{2}$ and cooled to $-78{ }^{\circ} \mathrm{C}$. A $1.0 \mathrm{M}$ DIBAL-H solution in toluene $(8.20 \mathrm{~mL}, 8.2$ mmol, 2 equiv) was slowly dripped down the side of the flask in order to pre-chill the solution. After 50 minutes, the solution was treated sequentially with pyridine $(1.00 \mathrm{~mL}$, 12.3 mmol, 3 equiv), DMAP (1.50 g, 12.3 mmol, 3 equiv), and acetic anhydride (2.32 $\mathrm{mL}, 24.6$ mmol, 6 equiv). The resulting solution was stirred overnight at $-78{ }^{\circ} \mathrm{C}$ and allowed to warm to $-10{ }^{\circ} \mathrm{C}$ over 3 hours. Upon warming, the reaction was quenched with $40 \mathrm{~mL}$ of aqueous ammonium chloride and $40 \mathrm{~mL}$ of saturated potassium tartrate. The heterogeneous mixture was stirred for approximately 2 hours. The aqueous layer was washed with $\mathrm{CH}_{2} \mathrm{Cl}_{2}(4 \times 20 \mathrm{~mL})$ and the combined organics were washed with a cold solution of $1 \mathrm{M}$ sodium bisulfate. The organics were further washed with saturated aqueous sodium bicarbonate $(3 \times 20 \mathrm{~mL})$, brine $(20 \mathrm{~mL})$, and then dried over $\mathrm{MgSO}_{4}$. Solvent was then removed in vacuo and the crude material was purified by flash chromatography using silica gel pretreated with triethylamine to afford $1.15 \mathrm{~g}(97 \%)$ of the title compound. Analytical data is representative of a 1:1 mixture of diastereomers: IR (neat) 3432, 3027, 2929, 2862, 1716, 1454, 1243, 1048, $700 \mathrm{~cm}^{-1} ;{ }^{1} \mathrm{H}$ NMR (500 $\left.\mathrm{MHz}, \mathrm{CDCl}_{3}\right) \delta 7.23(\mathrm{~m}, 5 \mathrm{H}), 5.95(\mathrm{dddd}, J=5.7,5.4,5.3,5.3 \mathrm{~Hz}, 1 \mathrm{H}), 5.80(\mathrm{~m}, 2 \mathrm{H})$, $5.12(\mathrm{~m}, 4 \mathrm{H}), 3.68$ (dddd, $\mathrm{J}=11.8,11.8,5.9,5.8,1 \mathrm{H}), 2.71(\mathrm{~m}, 2 \mathrm{H}), 2.49(\mathrm{~m}, 2 \mathrm{H})$, $2.32(\mathrm{~m}, 2 \mathrm{H}), 2.07(\mathrm{~s}, 1.5 \mathrm{H}), 2.04(\mathrm{~s}, 1.5 \mathrm{H}), 1.80(\mathrm{~m}, 2 \mathrm{H}) ;{ }^{13} \mathrm{C}$ NMR $(125 \mathrm{MHz}$, $\left.\mathrm{CDCl}_{3}\right) \delta 170.8,170.7,142.0,141.9,134.4,133.8,133.4,131.9,131.8,128.4,128.3$ $128.3,128.3,128.3,128.2,128.2,128.2,128.2,125.9,125.8,125.7,118.6,118.4,117.8$, $117.0,97.4,96.3,78.8,76.7,72.7,39.5,39.3,39.2,38.6,38.6,35.9,35.7,35.2,31.7$, 31.6, 31.2, 21.3, 21.2, 21.1 .

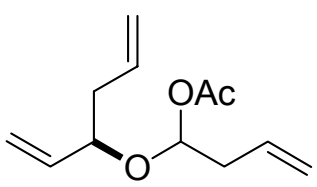




\section{$28 b$}

Acetoxy Ether 28b. Characterization is representative of a 1:1 mixture of diastereomers: IR (neat) 2983, 1739, 1432, 1238, $1126 \mathrm{~cm}^{-1} ;{ }^{1} \mathrm{H}$ NMR (400 MHz, $\left.\mathrm{CDCl}_{3}\right) \delta 5.93(\mathrm{t}, J=$ $5.5 \mathrm{~Hz}, 0.5 \mathrm{H}), 5.88$ (t, $J=5.4 \mathrm{~Hz}, 0.5 \mathrm{H}), 5.76(\mathrm{~m}, 3 \mathrm{H}), 5.15$ (m, $6 \mathrm{H}), 4.03$ (dq, $J=$ 12.8, $6.9 \mathrm{~Hz}, 1 \mathrm{H}), 2.40$ (m, $4 \mathrm{H}), 2.07$ (s, $1.5 \mathrm{H}), 2.01$ (s, $1.5 \mathrm{H}) ;{ }^{13} \mathrm{C}$ NMR $(125 \mathrm{MHz}$, $\left.\mathrm{CDCl}_{3}\right) \delta 170.7,170.5,138.5,137.1,134.0,133.8,131.8,131.7,118.5,118.4,118.3$, 117.6, 117.1, 115.5, 97.3, 95.2, 81.3, 79.0, 39.9, 39.7, 39.4, 39.2, 21.2, 15.3; LRMS (ESI) $m / z$ calcd for $\mathrm{C}_{12} \mathrm{H}_{18} \mathrm{O}_{3} \mathrm{Na}(\mathrm{M}+\mathrm{Na})^{+} 233$, found 233 .

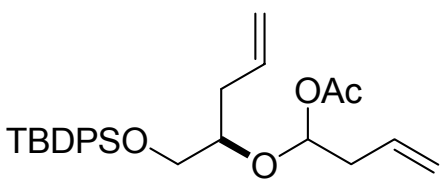

28c

Acetoxy Ether 28c. Characterization is representative of a 3:2 mixture of diastereomers: IR (neat) 2932, 1740, 1428, 1239, $1113 \mathrm{~cm}^{-1} ;{ }^{1} \mathrm{H}$ NMR (500 MHz, $\left.\mathrm{CDCl}_{3}\right) \delta 7.67$ (m, 4 H), $7.39(\mathrm{~m}, 6 \mathrm{H}), 6.07(\mathrm{t}, J=5.6 \mathrm{~Hz}, 0.4 \mathrm{H}), 5.91(\mathrm{t}, J=5.4 \mathrm{~Hz}, 0.6 \mathrm{H}), 5.75(\mathrm{~m}, 2 \mathrm{H})$, 5.05 (m, 4 H), 3.70 (m, 3 H), 2.41 (m, 4 H), 2.05 (s, 1.2 H), 1.87 (s, 1.8 H), 1.05 (s, 9 H); ${ }^{13} \mathrm{C}$ NMR $\left(125 \mathrm{MHz}, \mathrm{CDCl}_{3}\right) \delta 170.8,170.7,135.6,135.5,134.4,134.0,133.6,133.5$, 133.3, 131.9, 129.7 (2), 129.6, 127.7, 127.6, 118.4, 118.3, 117.8, 116.9, 97.8, 97.2, 79.6, 79.0, 66.0, 65.8, 65.2, 39.3, 36.3, 35.8, 26.8, 21.3, 21.2, 19.2, 19.1, 15.3; HRMS (ESI) $m$ / $z$ calcd for $\mathrm{C}_{27} \mathrm{H}_{36} \mathrm{O}_{4} \mathrm{Si}(\mathrm{M}+\mathrm{Na})^{+} 475.2281$, found 475.2299.

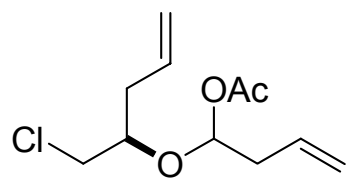


Acetoxy Ether 28d. Characterization is representative of a 3:2 mixture of diastereomers: IR (neat) 2959, 1740, 1643, 1434, $1238 \mathrm{~cm}^{-1} ;{ }^{1} \mathrm{H}$ NMR (500 MHz, $\left.\mathrm{CDCl}_{3}\right) \delta 5.95$ (t, $J=$ $5.5 \mathrm{~Hz}, 0.4 \mathrm{H}), 5.91$ (t, $J=5.6 \mathrm{~Hz}, 0.6 \mathrm{H}), 5.76(\mathrm{~m}, 2 \mathrm{H}), 5.14(\mathrm{~m}, 4 \mathrm{H}), 3.89(\mathrm{~m}, 1 \mathrm{H})$, $3.51(\mathrm{~m}, 2 \mathrm{H}), 2.44$ (m, $4 \mathrm{H}), 2.08$ (s, $1.8 \mathrm{H}), 2.06$ (s, $1.2 \mathrm{H}) ;{ }^{13} \mathrm{C}$ NMR $(125 \mathrm{MHz}$, $\left.\mathrm{CDCl}_{3}\right) \delta 170.9,170.8,133.2,132.6,131.6,131.5,118.9,118.7,118.6,118.1,97.2,96.9$, 78.3, 78.2, 45.8, 39.2, 39.1, 37.3, 36.6, 35.8, 21.3, 21.2; LRMS (ESI) $\mathrm{m} / \mathrm{z}$ calcd for $\mathrm{C}_{11} \mathrm{H}_{17} \mathrm{ClO}_{3} \mathrm{Na}(\mathrm{M}+\mathrm{H})^{+} 255$, found 255 .

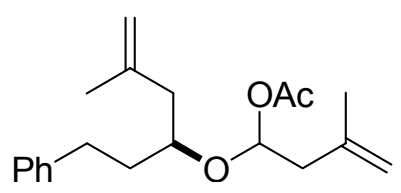

31

Acetoxy Ether 31. Characterization is representative of a 1:1 mixture of diastereomers: IR (neat) 2936, 1737, 1454, 1241, $1125 \mathrm{~cm}^{-1} ;{ }^{1} \mathrm{H} \mathrm{NMR}\left(500 \mathrm{MHz}, \mathrm{CDCl}_{3}\right) \delta 7.30$ (m, 2 H), 7.19 (m, 3 H), 6.06 (m, 1 H), 4.80 (m, 4 H), 3.77 (m, 1 H), 2.83 (ddd, $J=14.9,10.2$, $5.3 \mathrm{~Hz}, 0.5 \mathrm{H}$ ), 2.74 (ddd, $J=13.6,10.9,5.6 \mathrm{~Hz}, 0.5 \mathrm{H}), 2.62$ (m, 1H), 2.43 (m, $2 \mathrm{H}$ ), $2.34(\mathrm{~m}, 1 \mathrm{H}), 2.24(\mathrm{dd}, J=13.9,7.0 \mathrm{~Hz}, 0.5 \mathrm{H}), 2.17(\mathrm{dd}, J=14.1,7.3 \mathrm{~Hz}, 0.5 \mathrm{H}), 2.06$ (s, $1.5 \mathrm{H}), 2.03$ (s, $1.5 \mathrm{H}), 1.79(\mathrm{~m}, 8 \mathrm{H}) ;{ }^{13} \mathrm{C}$ NMR $\left(125 \mathrm{MHz}, \mathrm{CDCl}_{3}\right) \delta$ 171.0, 170.8, $142.3142 .2,142.1,142.0,140.1,140.0,128.4$ (2), 128.3 (2), 125.8, 125.7, 114.3, 114.1, 113.6, 113.0, 96.9, 95.9, 77.7, 76.0, 43.4, 43.3, 43.2 (2), 36.3, 35.6, 31.7, 31.2, 23.2, 23.1, 22.8, 22.6, 21.3, 21.2; HRMS (ESI) $m / z$ calcd for $\mathrm{C}_{20} \mathrm{H}_{28} \mathrm{O}_{3}(\mathrm{M}+\mathrm{Na})^{+} 339.1936$, found 339.1929 . 


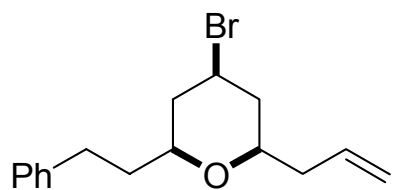

26

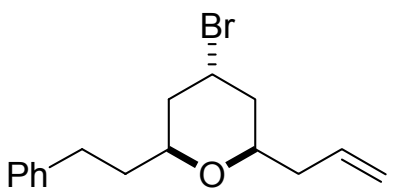

27

Tetrahydropyrans 26 and $27 .{ }^{3}$ A solution of desired concentration $\mathrm{SnBr}_{4}$ in dichloromethane was prepared and cooled to $-78{ }^{\circ} \mathrm{C}$. Acetoxy ether $25(0.050 \mathrm{~g}, 0.18$ mmol, 1.0 equiv) was dissolved in $0.60 \mathrm{~mL}$ of $\mathrm{CH}_{2} \mathrm{Cl}_{2}$, cooled to $-78^{\circ} \mathrm{C}$, and then slowly added dropwise to a solution of the Lewis acid. The solution was allowed to stir for 1 hour, whereupon the excess Lewis acid was quenched with saturated aqueous sodium bicarbonate. The aqueous layer was washed with $\mathrm{CH}_{2} \mathrm{Cl}_{2}(5 \mathrm{~mL} \times 3)$ and the combined organic phases were washed with brine $(3 \mathrm{~mL})$, dried over $\mathrm{MgSO}_{4}$, and concentrated in vacuo. Flash chromatography (0-1\% ether/hexanes) afforded $33 \mathrm{mg}$ of $26(60 \%)$ and 18 $\mathrm{mg}$ of $27(33 \%)$ as a clear oil. Major isomer $26:{ }^{1} \mathrm{H} \mathrm{NMR}\left(500 \mathrm{MHz}, \mathrm{CDCl}_{3}\right) \delta 7.24(\mathrm{~m}$, 5H), 5.92 (dddd, $J=17.4,10.2,7.0,6.8,1 \mathrm{H}), 5.13$ (m, $2 \mathrm{H}), 4.12(\mathrm{tt}, J=12.0,12.0,4.5$, $4.5 \mathrm{~Hz}, 1 \mathrm{H}$ ), 3.33 (dddd, $J=11.0,7.2,5.5,1.8 \mathrm{~Hz}, 1 \mathrm{H}$ ), 3.25 (dddd, $J=10.9,8.7,4.1$, 1.8, $1 \mathrm{H}$ ), 2.79 (ddd, $J=13.9,8.9,5.3 \mathrm{~Hz}, 1 \mathrm{H}), 2.70$ (m, $1 \mathrm{H}$ ), 2.39 (ddd, 14.1, 6.8, 6.7 $\mathrm{Hz}, 1 \mathrm{H}), 2.23$ (m, $3 \mathrm{H}), 1.90$ (dtd, $J=13.8,8.7,5.4 \mathrm{~Hz}, 1 \mathrm{H}), 1.73$ (m, $3 \mathrm{H}) ;{ }^{13} \mathrm{C}$ NMR $\left(125 \mathrm{MHz}, \mathrm{CDCl}_{3}\right) \delta 141.7,134.2,128.5,128.3,125.8,117.2,76.7,76.2,46.8,43.2$, 42.9, 40.2, 37.3, 31.4; IR (neat) 3077, 3026, 2849, 1642, 1082, $700 \mathrm{~cm}^{-1}$; HRMS (CI/ $\left./ \mathrm{NH}_{3}\right) \mathrm{m} / z$ calcd for $\mathrm{C}_{16} \mathrm{H}_{22} \mathrm{BrO}(\mathrm{M})^{+}$308.0776, found 308.0776. Anal. Calcd for $\mathrm{C}_{16} \mathrm{H}_{21} \mathrm{BrO}: \mathrm{C}, 62.14 ; \mathrm{H}, 6.84$. Found: $\mathrm{C}, 62.33 ; \mathrm{H}, 6.76$. Minor Isomer 27: ${ }^{1} \mathrm{H}$ NMR $\left(500 \mathrm{MHz}, \mathrm{CDCl}_{3}\right) \delta 7.25(\mathrm{~m}, 5 \mathrm{H}), 5.90(\mathrm{dddd}, J=17.1,10.3,7.3,6.6 \mathrm{~Hz}, 1 \mathrm{H}), 5.12(\mathrm{~m}$, 2 H), 4.74 (dddd, $J=3.0,3.0,3.0,3.0 \mathrm{~Hz}, 1 \mathrm{H}$ ), 3.90 (m, 2 H), 2.84 (ddd, $J=13.9,9.9$, $5.3 \mathrm{~Hz}, 1 \mathrm{H}), 2.69$ (ddd, $J=13.7,9.8,6.7 \mathrm{~Hz}, 1 \mathrm{H}), 2.38$ (ddd, $J=14.1,6.9,6.9 \mathrm{~Hz}, 1 \mathrm{H}$ ), 2.25 (ddd, $J=14.2,7.2,5.9,1 \mathrm{H}), 2.00$ (m, $2 \mathrm{H}), 1.87$ (dddd, $J=13.8,9.8,8.6,5.3 \mathrm{~Hz}, 1$ $\mathrm{H}), 1.73(\mathrm{~m}, 3 \mathrm{H}) ;{ }^{13} \mathrm{C}$ NMR $\left(125 \mathrm{MHz}, \mathrm{CDCl}_{3}\right) \delta 142.1,134.6,128.4,128.3,125.7$,

\footnotetext{
${ }^{3}$ Jaber, J. J.; Mitsui, K.; Rychnovsky, S. D. J. Org. Chem. 2001, 66, 4679.
} 
116.9, 71.7, 71.4, 50.7, 40.0, 39.6, 39.2, 37.4, 31.7; IR (neat) 3026, 2948, 1642, 1496, 1077, $699 \mathrm{~cm}^{-1}$; HRMS $\left(\mathrm{CI} / \mathrm{NH}_{3}\right) \mathrm{m} / z$ calcd for $\mathrm{C}_{16} \mathrm{H}_{22} \mathrm{BrO}(\mathrm{M}+\mathrm{H})^{+}$309.0856, found 309.0810. Anal. Calcd for $\mathrm{C}_{16} \mathrm{H}_{21} \mathrm{BrO}$ : C, 62.14; H, 6.84. Found: $\mathrm{C}, 62.13 ; \mathrm{H}, 6.65$.

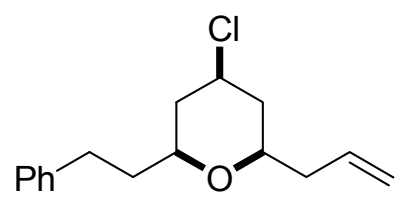

59

Tetrahydropyran 59. IR (neat) 2926, 1451, $1083 \mathrm{~cm}^{-1} ;{ }^{1} \mathrm{H}$ NMR (400 MHz, $\left.\mathrm{CDCl}_{3}\right) \delta$ 7.27 (m, 2 H), 7.17 (m, 3 H), 5.86 (ddt, $J=17.2,10.2,7.2 \mathrm{~Hz}, 1 \mathrm{H}), 5.10(\mathrm{~m}, 2 \mathrm{H}), 3.95$ (tt, $J=11.8,4.5 \mathrm{~Hz}, 1 \mathrm{H}$ ), 3.30 (dddd, $J=12.8,7.3,5.5,1.8 \mathrm{~Hz}, 1 \mathrm{H}$ ), 3.22 (dddd, $J=$ 10.8, 8.6, 4.1, $1.8 \mathrm{~Hz}, 1 \mathrm{H}), 2.73$ (m $2 \mathrm{H}), 2.37$ (dt, $J=14.0,6.7 \mathrm{~Hz}, 1 \mathrm{H}), 2.22$ (dt, $J=$ 13.3, $7.1 \mathrm{~Hz}, 1 \mathrm{H}), 2.10$ (m, $2 \mathrm{H}), 1.87$ (dtd, $J=13.98 .7,5.4 \mathrm{~Hz}, 1 \mathrm{H}$ ), 1.70 (dddd, $J=$ 13.4, 9.0, 7.6, 4.2 Hz, $1 \mathrm{H}), 1.52(\mathrm{~m}, 2 \mathrm{H}) ;{ }^{13} \mathrm{C} \mathrm{NMR}\left(125 \mathrm{MHz}, \mathrm{CDCl}_{3}\right) \delta 141.8,134.3$, $128.5,128.3,125.8,117.2,76.1,75.4,55.8,42.4,42.0,40.2,37.3,31.5$; HRMS (ESI) $m /$ $z$ calcd for $\mathrm{C}_{16} \mathrm{H}_{21} \mathrm{ClO}(\mathrm{M}+\mathrm{Na})^{+} 287.1179$, found 287.1174. Anal. Calcd for $\mathrm{C}_{16} \mathrm{H}_{21} \mathrm{ClO}$ : C, 72.57; H, 7.99. Found: C, 72.36; H, 8.15.

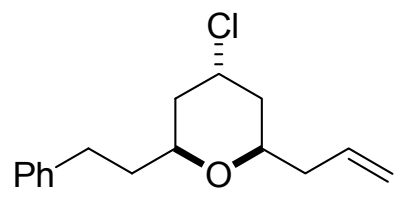

60

Tetrahydropyran 60. IR (neat) 2949, 1432, 1065, $699 \mathrm{~cm}^{-1} ;{ }^{1} \mathrm{H}$ NMR $(400 \mathrm{MHz}$, $\left.\mathrm{CDCl}_{3}\right) \delta 7.28(\mathrm{~m}, 2 \mathrm{H}), 7.19(\mathrm{~m}, 3 \mathrm{H}), 5.88(\mathrm{ddt}, J=17.1,10.2,7.2 \mathrm{~Hz}, 1 \mathrm{H}), 5.10(\mathrm{~m}, 2$ H), 4.58 (quin, $J=2.97 \mathrm{~Hz}, 1 \mathrm{H}$ ), $2.82(\mathrm{ddd}, J=14.0,9.9,5.4 \mathrm{~Hz}, 1 \mathrm{H}$ ), 2.67 (ddd, $J=$ 13.8, 9.7, $6.8 \mathrm{~Hz}, 1 \mathrm{H}), 2.35$ (dt, $J=14.0,6.8 \mathrm{~Hz}, 1 \mathrm{H}), 2.18(\mathrm{dt}, J=13.1,6.6 \mathrm{~Hz}, 1 \mathrm{H})$, $1.87(\mathrm{~m}, 3 \mathrm{H}), 1.69(\mathrm{~m}, 3 \mathrm{H}) ;{ }^{13} \mathrm{C} \mathrm{NMR}\left(125 \mathrm{MHz}, \mathrm{CDCl}_{3}\right) \delta 142.2,134.6,128.5,128.3$, 
125.7, 116.9, 71.1, 70.7, 57.0, 40.2, 39.2, 38.8, 37.5, 31.7; HRMS (ESI) $m / z$ calcd for $\mathrm{C}_{16} \mathrm{H}_{21} \mathrm{ClO}(\mathrm{M}+\mathrm{Na})^{+}$287.1179, found 287.1174.

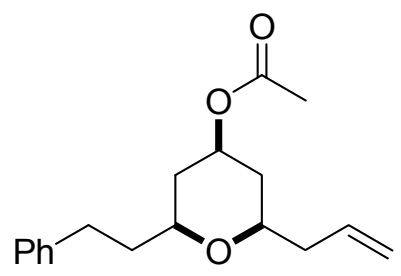

61

Tetrahydropyran $61 .^{2} \alpha$-Acetoxy ether 25 (50 mg, $0.18 \mathrm{mmol}, 1$ equiv) was dissolved in $4 \mathrm{~mL}$ hexanes and cooled to $0{ }^{\circ} \mathrm{C}$. To this was added a premixed solution of $\mathrm{BF}_{3} \cdot \mathrm{OEt}_{2}$ (70 $\mu \mathrm{L}, 0.55$ mmol, 3 equiv) and $\mathrm{AcOH}(90 \mu \mathrm{L}, 1.6 \mathrm{mmol}, 8.6$ equiv) in $2 \mathrm{~mL}$ of hexanes. The mixture was stirred for one hour whereupon it was diluted with saturated aqueous sodium bicarbonate, washed with brine, and dried over $\mathrm{MgSO}_{4}$. Solvent was removed in vacuo and the crude product was purified by flash chromatography $(0-5 \%$ ether/hexane) to afford a $26 \mathrm{mg}(52 \%)$ of a clear oil: ${ }^{1} \mathrm{H} \mathrm{NMR}\left(500 \mathrm{MHz}, \mathrm{CDCl}_{3}\right) \delta 7.23$ (m, 5 H), 5.88 (dddd, $\mathrm{J}=17.1,10.1,7.2,7.0,1 \mathrm{H}$ ), 5.09 (m, 2 H), 4.85 (dddd, $\mathrm{J}=11.3$, 11.2, 4.8, 4.8 Hz, $1 \mathrm{H}$ ), 3.39 (dddd, $\mathrm{J}=10.9,7.4,5.6,1.9 \mathrm{~Hz}, 1 \mathrm{H}$ ), 3.31 (ddd, J = 11.1, 8.7, 4.1, 1.8 Hz, $1 \mathrm{H}$ ), 2.79 (ddd, $\mathrm{J}=13.8,9.2,5.3 \mathrm{~Hz}, 1 \mathrm{H}), 2.69$ (ddd, $\mathrm{J}=13.8,8.8,7.6$ Hz, $1 \mathrm{H}), 2.39$ (ddd, J = 14.1, 6.8, $6.8 \mathrm{~Hz}, 1 \mathrm{H}), 2.23$ (ddd, J = 13.0, 7.2, 7.2 Hz, $1 \mathrm{H}$ ), 2.03 (s, $3 \mathrm{H}), 1.93$ (m, $3 \mathrm{H}), 1.71$ (dddd, $\mathrm{J}=13.1,9.9,7.5,4.2,1 \mathrm{H}), 1.26(\mathrm{~m}, 2 \mathrm{H}) ;{ }^{13} \mathrm{C}$ NMR (125 MHz, $\left.\mathrm{CDCl}_{3}\right) \delta 170.5,141.9,134.5,128.5,128.3,125.8,117.0,74.9,74.2$, 70.5, 40.4, 37.5, 37.3, 36.9, 31.6, 21.3; IR (neat) 2926, 1741, 1366, 1242, 1029, $701 \mathrm{~cm}^{-1}$; HRMS $\left(\mathrm{CI} / \mathrm{NH}_{3}\right) m / z$ calcd for $\mathrm{C}_{18} \mathrm{H}_{25} \mathrm{O}_{3}(\mathrm{M}+\mathrm{H})^{+}$289.1805, found 289.1765. Anal. Calcd for $\mathrm{C}_{18} \mathrm{H}_{24} \mathrm{O}_{3}$ : C, 74.97; H, 8.39. Found: C, 75.03; H, 8.27.

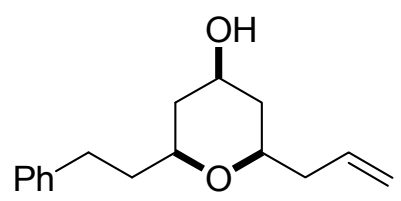


Tetrahydropyran 62. ${ }^{4}$ IR (neat) $3435,2900,2864,1254,1104,1077,738,699 \mathrm{~cm}^{-1} ;{ }^{1} \mathrm{H}$ NMR (400 MHz, $\left.\mathrm{CDCl}_{3}\right) \delta 7.23$ (m, $5 \mathrm{H}$ ), 5.89 (dddd, $J=16.8,10.0,7.4,7.1,1 \mathrm{H}$ ), 5.09 (m, $2 \mathrm{H}), 3.75$ (m, $1 \mathrm{H}), 3.31$ (dtd, $J=11.2,6.4,1.9 \mathrm{~Hz}, 1 \mathrm{H}), 3.23$ (dddd, $J=10.9,8.8$, 4.2, $1.9 \mathrm{~Hz}, 1 \mathrm{H}$ ), 2.79 (ddd, $J=14.0,8.9,5.4 \mathrm{~Hz}, 1 \mathrm{H}$ ), 2.69 (ddd, $J=14.0,8.2,7.9 \mathrm{~Hz}$, $1 \mathrm{H}), 2.39$ (dt, $J=14.2,6.8,6.8 \mathrm{~Hz}, 1 \mathrm{H}), 2.23$ (ddd, $J=14.2,7.2,6.0 \mathrm{~Hz}, 1 \mathrm{H}$ ), 1.93 (m, $3 \mathrm{H}), 1.71$ (dddd, $J=13.4,9.3,7.1,4.0,1 \mathrm{H}), 1.54$ (s, $1 \mathrm{H}), 1.16$ (m, $2 \mathrm{H}) ;{ }^{13} \mathrm{C}$ NMR $(125$ $\left.\mathrm{MHz}, \mathrm{CDCl}_{3}\right) \delta 142.1,134.8,128.5,128.2,125.7,116.8,75.0,74.2,68.2,41.5,40.8$, 40.5, 37.5, 31.7; HRMS (ESI) $m / z$ calcd for $\mathrm{C}_{16} \mathrm{H}_{22} \mathrm{O}_{2}(\mathrm{M}+\mathrm{Na})^{+} 269.1518$, found 269.1518. Anal. Calcd for $\mathrm{C}_{16} \mathrm{H}_{22} \mathrm{O}_{2}$ : C, 78.01; H, 9.00. Found: C, 77.89; H, 8.81.

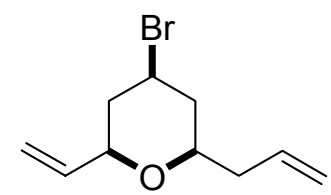

29b

Tetrahydropyran 29b. IR (neat) 2958, 1643, 1445, 1076, $920 \mathrm{~cm}^{-1} ;{ }^{1} \mathrm{H}$ NMR (500 $\left.\mathrm{MHz}, \mathrm{CDCl}_{3}\right) \delta 5.82(\mathrm{~m}, 2 \mathrm{H}), 5.27(\mathrm{dt}, J=17.3,1.3 \mathrm{~Hz}, 1 \mathrm{H}), 5.10(\mathrm{~m}, 3 \mathrm{H}), 4.17$ (tt, $J=$ 12.1, $4.4 \mathrm{~Hz}, 1 \mathrm{H}), 3.84$ (ddd, $J=11.2,5.4,1.6 \mathrm{~Hz}, 1 \mathrm{H}), 3.41$ (dtd, $J=11.0,6.2,1.9 \mathrm{~Hz}$, $1 \mathrm{H}), 2.38(\mathrm{dt}, J=14.1,6.4 \mathrm{~Hz}, 1 \mathrm{H}), 2.25(\mathrm{~m}, 3 \mathrm{H}), 1.80$ (q, $J=11.9 \mathrm{~Hz}, 1 \mathrm{H}), 1.71$ (q, $J$ $=12.1 \mathrm{~Hz}, 1 \mathrm{H}) ;{ }^{13} \mathrm{C} \mathrm{NMR}\left(125 \mathrm{MHz}, \mathrm{CDCl}_{3}\right) \delta 137.4,133.9,117.5,115.7,77.9,76.8$, 46.3, 42.9, 42.4, 40.2; LRMS (ESI) $m / z$ calcd for $\mathrm{C}_{10} \mathrm{H}_{15} \mathrm{BrONa}(\mathrm{M}+\mathrm{Na})^{+} 253$, found 253.

\footnotetext{
${ }^{4}$ Barry, C. St. J.; Crosby, S. R.; Harding, J. R.; Hughes, R. A.; King, C. D.; Parker, G. D.; Willis, C. L. Org. Lett. 2003, 5, 2429.
} 


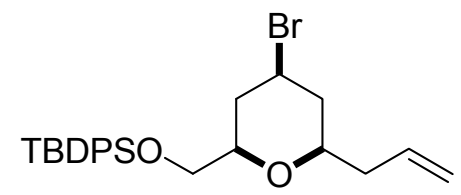

29c

Tetrahydropyran 29c. IR (neat) 2930, 1642, 1428, 1113, $702 \mathrm{~cm}^{-1} ;{ }^{1} \mathrm{H}$ NMR $(500 \mathrm{MHz}$, $\left.\mathrm{CDCl}_{3}\right) \delta 7.67(\mathrm{~m}, 4 \mathrm{H}), 7.39(\mathrm{~m}, 6 \mathrm{H}), 5.80(\mathrm{ddt}, J=17.2,10.2,7.1 \mathrm{~Hz}, 1 \mathrm{H}), 5.06(\mathrm{~m}, 2$ H), 4.14 (tt, $J=12.0,4.4 \mathrm{~Hz}, 1 \mathrm{H}), 3.72(\mathrm{dd}, J=10.4,5.3 \mathrm{~Hz}, 1 \mathrm{H}), 3.58$ (dd, $J=10.4$, $5.4 \mathrm{~Hz}, 1 \mathrm{H}), 3.46(\mathrm{dtd}, J=11.2,5.3,1.8 \mathrm{~Hz}, 1 \mathrm{H}), 3.34(\mathrm{dtd}, J=10.9,6.1,1.9,1 \mathrm{H}), 2.25$ (m, $4 \mathrm{H}), 1.72(\mathrm{~m}, 2 \mathrm{H}), 1.05(\mathrm{~s}, 9 \mathrm{H}) ;{ }^{13} \mathrm{C}$ NMR $\left(125 \mathrm{MHz}, \mathrm{CDCl}_{3}\right) \delta$ 135.7, 135.6, 134.0, 133.6, 133.5, 129.7, 129.6, 127.6 (2), 117.3, 78.0, 76.8, 66.5, 46.8, 42.8, 40.1, 39.9, 26.8, 19.3; HRMS (ESI) $m / z$ calcd for $\mathrm{C}_{25} \mathrm{H}_{33} \mathrm{O}_{2} \mathrm{BrSi}(\mathrm{M}+\mathrm{Na})^{+}$495.1331, found 495.1331 .

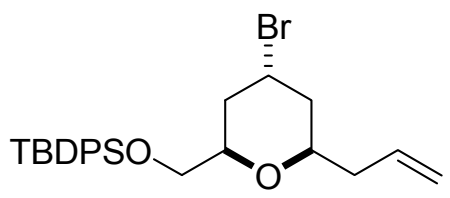

30c

Tetrahydropyran 30c. IR (neat) 2930, 1428, 1113, $702 \mathrm{~cm}^{-1}$; ${ }^{1} \mathrm{H}$ NMR (500 MHz, $\left.\mathrm{CDCl}_{3}\right) \delta 7.69$ (m, $\left.4 \mathrm{H}\right), 7.39$ (m, $\left.6 \mathrm{H}\right), 5.84$ (ddt, $\left.J=17.1,10.2,6.9,1 \mathrm{H}\right), 5.06(\mathrm{~m}, 2 \mathrm{H})$, 4.76 (quin, $J=3.0 \mathrm{~Hz}, 1 \mathrm{H}), 4.02(\mathrm{dtd}, J=10.9,5.0,1.9 \mathrm{~Hz}, 1 \mathrm{H}), 3.93(\mathrm{dtd}, J=11.5$, 6.2, $1.7 \mathrm{~Hz}, 1 \mathrm{H}), 3.74(\mathrm{dd}, J=10.6,5.2 \mathrm{~Hz}, 1 \mathrm{H}), 3.64(\mathrm{dd}, J=10.6,5.0 \mathrm{~Hz}, 1 \mathrm{H}), 2.31$ (dt, $J=14.3,6.6 \mathrm{~Hz}, 1 \mathrm{H}), 2.19(\mathrm{dt}, J=13.2,7.1 \mathrm{~Hz}, 1 \mathrm{H}), 2.04(\mathrm{dq}, J=14.5,2.3 \mathrm{~Hz}, 1$ H), 1.98 (dq, $J=14.6,2.3 \mathrm{~Hz}, 1 \mathrm{H}), 1.83$ (ddd, $J=14.4,11.0,3.4 \mathrm{~Hz}, 1 \mathrm{H}$ ), 1.71 (ddd, $J$ $=14.3,10.8,3.3 \mathrm{~Hz}, 1 \mathrm{H}), 1.05(\mathrm{~s}, 9 \mathrm{H}) ;{ }^{13} \mathrm{C} \mathrm{NMR}\left(125 \mathrm{MHz}, \mathrm{CDCl}_{3}\right) \delta$ 135.7, 135.6, 134.5 (2), 133.7, 133.6, 129.6, 127.6 (2), 116.9, 72.9, 71.5, 66.7, 50.6, 39.9, 39.0, 36.1, 
26.8, 19.3; HRMS (ESI) $m / z$ calcd for $\mathrm{C}_{25} \mathrm{H}_{33} \mathrm{O}_{2} \mathrm{BrSi}(\mathrm{M}+\mathrm{Na})^{+}$495.1331, found 495.1339. Anal. Calcd for $\mathrm{C}_{25} \mathrm{H}_{33} \mathrm{BrO}_{2} \mathrm{Si}$ : C, 63.41; H, 7.02. Found: $\mathrm{C}, 63.09 ; \mathrm{H}, 7.16$

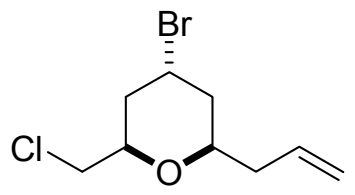

30d

Tetrahydropyran 30d. $[\alpha]^{24}-8.2^{\circ}\left(c 1.1, \mathrm{CHCl}_{3}\right)$; IR (neat) $2899,1642,1430,1067$ $\mathrm{cm}^{-1} ;{ }^{1} \mathrm{H}$ NMR (500 MHz, $\mathrm{CDCl}_{3}$ ) $\delta 5.84$ (dddd, $\left.J=17.1,10.2,7.4,6.6 \mathrm{~Hz}, 1 \mathrm{H}\right), 5.09$ (m, $2 \mathrm{H}$ ), 4.76 (quin, $J=3.1 \mathrm{~Hz}, 1 \mathrm{H}$ ), 4.14 (dtd, $J=10.6,5.2,1.7 \mathrm{~Hz}, 1 \mathrm{H}), 3.99$ (dtd, $J=$ 10.9, 6.3, $1.8 \mathrm{~Hz}, 1 \mathrm{H}), 3.55$ (m, $2 \mathrm{H}), 2.37$ (m, $1 \mathrm{H}), 2.23$ (dt, $J=13.5,6.7 \mathrm{~Hz}, 1 \mathrm{H}), 2.07$ (dq, $J=13.8,2.0 \mathrm{~Hz}, 1 \mathrm{H}), 2.00(\mathrm{dq}, J=14.6,2.0 \mathrm{~Hz}, 1 \mathrm{H}), 1.87$ (ddd, $J=4.4,10.9,3.4$ $\mathrm{Hz}, 1 \mathrm{H}), 1.74$ (ddd, $J=14.4,10.8,3.4 \mathrm{~Hz}, 1 \mathrm{H}) ;{ }^{13} \mathrm{C} \mathrm{NMR}\left(125 \mathrm{MHz}, \mathrm{CDCl}_{3}\right) \delta 134.0$, 117.2, 72.0, 71.9, 49.5, 46.8, 39.7, 38.6, 36.9; LRMS $\left(\mathrm{CI} / \mathrm{NH}_{3}\right) \mathrm{m} / z$ calcd for $\mathrm{C}_{9} \mathrm{H}_{15} \mathrm{BrClO}(\mathrm{M}+\mathrm{H})^{+} 252$, found 252 .

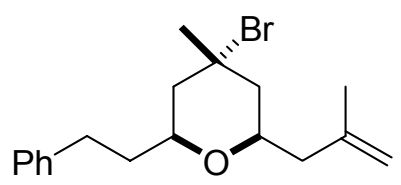

32

Tetrahydropyran 32. $[\alpha]^{24}-9.3^{\circ}\left(c 1.1, \mathrm{CHCl}_{3}\right)$; IR (neat) $2938,1454,1374,1104$, $699 \mathrm{~cm}^{-1} ;{ }^{1} \mathrm{H}$ NMR (500 MHz, $\left.\mathrm{CDCl}_{3}\right) \delta 7.29$ (m, $\left.2 \mathrm{H}\right), 7.21$ (m, $\left.3 \mathrm{H}\right), 4.84(\mathrm{~s}, 1 \mathrm{H}), 4.80$ (s, $1 \mathrm{H}$ ), 3.96 (dddd, $J=10.4,7.4,6.0,1.7 \mathrm{~Hz}, 1 \mathrm{H}$ ), 3.81 (dddd, $J=10.5,8.7,4.2,1.8$ $\mathrm{Hz}, 1 \mathrm{H}), 2.85$ (ddd, $J=13.7,10.3,5.3 \mathrm{~Hz}, 1 \mathrm{H}), 2.69$ (ddd, $J=13.8,10.0,6.5 \mathrm{~Hz}, 1 \mathrm{H}$ ), $2.37(\mathrm{dd}, J=14.1,7.2 \mathrm{~Hz}, 1 \mathrm{H}), 2.17(\mathrm{dd}, J=14.1,6.0 \mathrm{~Hz}, 1 \mathrm{H}), 2.06$ (m, $2 \mathrm{H}), 1.85$ (m, 7 H), $1.72(\mathrm{~m}, 1 \mathrm{H}), 1.34$ (dd, $J=14.5,10.6 \mathrm{~Hz}, 1 \mathrm{H}), 1.28(\mathrm{dd}, J=14.6,10.6 \mathrm{~Hz}, 1 \mathrm{H})$; ${ }^{13} \mathrm{C}$ NMR $\left(125 \mathrm{MHz}, \mathrm{CDCl}_{3}\right) \delta 142.4,142.2,128.4,128.3,125.7,112.5,73.5,72.6,67.7$, 
47.5, 47.2, 43.8, 37.3, 35.9, 31.9, 22.9; Anal. Calcd for $\mathrm{C}_{18} \mathrm{H}_{25} \mathrm{BrO}$ : C, 64.10; H, 7.47. Found: $\mathrm{C}, 64.35 ; \mathrm{H}, 7.25$.

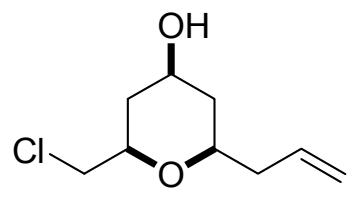

cis 52

Cis Tetrahydropyran 52. ${ }^{4}$ IR (neat) 3365, 2944, 1642, 1371, $1040 \mathrm{~cm}^{-1} ;{ }^{1} \mathrm{H}$ NMR (500 $\mathrm{MHz}, \mathrm{CDCl}_{3}$ ) $\delta 5.84$ (ddt, $J=17.1,10.3,6.9 \mathrm{~Hz}, 1 \mathrm{H}$ ), 5.08 (m, $2 \mathrm{H}$ ), 3.84 (quin, $J=5.8$ $\mathrm{Hz}, 1 \mathrm{H}), 3.55$ (m, $3 \mathrm{H}), 3.41$ (dtd, $J=10.9,6.3,1.9 \mathrm{~Hz}, 1 \mathrm{H}), 2.41$ (dt, $J=14.1,6.5 \mathrm{~Hz}$, $1 \mathrm{H}), 2.25(\mathrm{dt}, J=13.9,6.8 \mathrm{~Hz}, 1 \mathrm{H}), 2.11(\mathrm{ddt}, J=12.2,4.7,2.4 \mathrm{~Hz}, 1 \mathrm{H}), 1.99$ (ddt, $J=$ 12.4, 4.2, $2.3 \mathrm{~Hz}, 1 \mathrm{H}), 1.60(\mathrm{~s}, 1 \mathrm{H}), 1.22(\mathrm{~m}, 2 \mathrm{H}) ;{ }^{13} \mathrm{C} \mathrm{NMR}\left(125 \mathrm{MHz}, \mathrm{CDCl}_{3}\right) \delta$ 134.2, 117.2, 75.4, 75.3, 67.8, 46.8, 40.3, 40.1, 38.4; HRMS (ESI) $m / z$ calcd for $\mathrm{C}_{9} \mathrm{H}_{15} \mathrm{O}_{2} \mathrm{Cl}(\mathrm{M}+\mathrm{Na})^{+} 213.0658$, found 213.0659 .

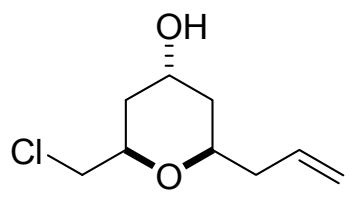

trans 52

Trans Tetrahydropyran 52. ${ }^{4}$ IR (neat) 3364, 2955, 1642, 1430, $1067 \mathrm{~cm}^{-1} ;{ }^{1} \mathrm{H}$ NMR $\left(500 \mathrm{MHz}, \mathrm{CDCl}_{3}\right) \delta 5.86$ (ddt, $\left.J=17.2,10.2,7.0 \mathrm{~Hz}, 1 \mathrm{H}\right), 5.07(\mathrm{~m}, 2 \mathrm{H}), 4.33(\mathrm{~m}, 1 \mathrm{H})$, $4.03(\mathrm{dtd}, J=11.5,5.5,2.1 \mathrm{~Hz}, 1 \mathrm{H}), 3.89(\mathrm{dtd}, J=10.9,6.3,2.0 \mathrm{~Hz}, 1 \mathrm{H}), 3.52(\mathrm{~m}, 2 \mathrm{H})$, $2.35(\mathrm{dt}, J=13.0,6.5 \mathrm{~Hz}, 1 \mathrm{H}), 2.19$ (dt, $J=14.4,7.2 \mathrm{~Hz}, 1 \mathrm{H}), 1.78$ (dq, $J=13.8,2.4$ $\mathrm{Hz}, 1 \mathrm{H}$ ), 1.70 (dq, $J=14.0,2.6 \mathrm{~Hz}, 1 \mathrm{H}), 1.58$ (ddd, $J=14.3,11.8,2.8,1 \mathrm{H}), 1.49$ (ddd, $J=14.3,11.7,2.8 \mathrm{~Hz}, 1 \mathrm{H}), 1.40($ br s, $1 \mathrm{H}) ;{ }^{13} \mathrm{C} \mathrm{NMR}\left(125 \mathrm{MHz}, \mathrm{CDCl}_{3}\right) \delta 134.5$, 
116.9, 71.5, 64.4, 47.5, 40.3, 40.1, 37.8, 35.9; HRMS (ESI) $m / z$ calcd for $\mathrm{C}_{9} \mathrm{H}_{15} \mathrm{ClO}_{2}(\mathrm{M}$ $+\mathrm{Na})^{+} 213.0658$, found 213.0667 .

\section{$\underline{\text { Analysis of Enantiomeric Excess }}$}

Enantiomeric excess of the following compounds was assessed by Hewlett Packard Series 1100 HPLC with Chiracel OD-H column. Listed below are conditions used to separate racemic samples. The solvent ratio is listed as a ratio of hexane to isopropanol.

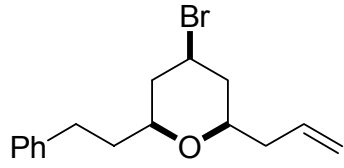

Solvent ratio: $99.6: 0.4$ Flow Rate: $0.9 \mathrm{ml} / \mathrm{min}$ Retention Times: 5.95, 6.30 min

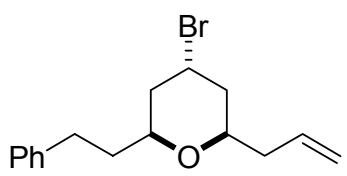

Solvent ratio: $99.6: 0.4$ Flow Rate: $0.9 \mathrm{ml} / \mathrm{min}$

Retention Times: 5.73, $6.30 \mathrm{~min}$<smiles>C=CCC1CC(Cl)CC(CCc2ccccc2)O1</smiles>

Solvent ratio: $99.9: 0.1$

Flow Rate: $1.1 \mathrm{ml} / \mathrm{min}$

Retention Times: 8.50, $9.08 \mathrm{~min}$<smiles>C=CCC1C[C@@H](Cl)CC(CCc2ccccc2)O1</smiles>

Solvent ratio: $99.9: 0.1$

Flow Rate: $1.1 \mathrm{ml} / \mathrm{min}$

Retention Times: 7.76, $10.12 \mathrm{~min}$

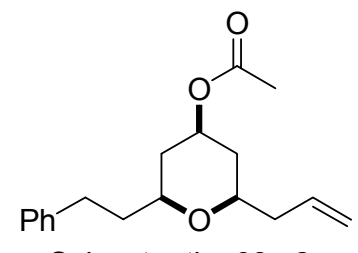

Solvent ratio: $98: 2$

Flow Rate: $0.9 \mathrm{ml} / \mathrm{min}$

Retention Times: 7.91, $8.75 \mathrm{~min}$<smiles>C=CC[C@@H]1CC(O)CC(CCc2ccccc2)O1</smiles>

Solvent ratio: $98: 2$

Flow Rate: $1.1 \mathrm{ml} / \mathrm{in}$

Retention Times: 11.69, 12.60 min

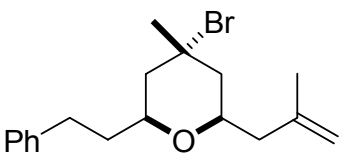

Solvent ratio: $99.9: 0.1$

Flow Rate: $1.1 \mathrm{ml} / \mathrm{min}$

Retention Times: 16.23, 19.51<smiles></smiles>

Solvent ratio: $99: 1$

Flow Rate: $1.1 \mathrm{ml} / \mathrm{min}$

Retention Times: 9.21, $10.81 \mathrm{~min}$

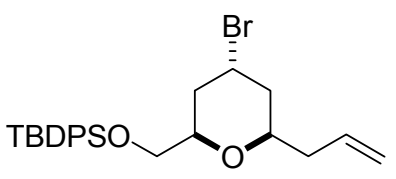

Solvent ratio: $99: 1$

Flow Rate: $1.1 \mathrm{ml} / \mathrm{min}$

Retention Times: 7.05, $8.24 \mathrm{~min}$ 
Enantiomeric excess of the following compounds was assessed by Hewlett Packard 5890 Series II GC with a $\gamma$-TA Chiraldex column. Listed below are conditions used to separate racemic samples.

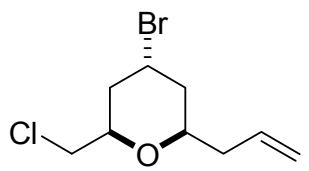

Gradient: $50{ }^{\circ} \mathrm{C}$ to $85^{\circ} \mathrm{C}: 0.5 \mathrm{deg} / \mathrm{min}$ Retention Times: $120.86,123.47 \mathrm{~min}$<smiles>C=CCC1CC(Br)CC(CCl)O1</smiles>

Gradient: $50^{\circ} \mathrm{C}$ to $85^{\circ} \mathrm{C}$ : $0.5 \mathrm{deg} / \mathrm{min}$ Retention times: $140.36,142.96 \mathrm{~min}$

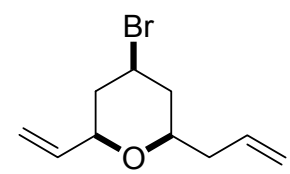

Gradient: $50{ }^{\circ} \mathrm{C}$ to $95^{\circ} \mathrm{C}: 1.0 \mathrm{deg} / \mathrm{min}$ Retention times: $47.54,48.26 \mathrm{~min}$

\section{Optimized Geometries}

Geometry optimizations were performed with B3LYP/6-31G* as implemented in Gaussian 03. Minima were characterized by their vibrational frequencies. Reported energies include unscaled zero-point corrections.

chloromethyl cation 43 (open form 1)

b3lyp/6-31g(d)<smiles>C=CCC(CCl)O[C-]C</smiles>

H $-2.3748638436,1.6321889315,-0.2979671178$

C $-2.2632630097,1.5934161715,0.7839584843$

C $0.7421652263,1.6890237676,0.5522556372$

C $-1.7355804697,0.5238617212,1.4012545899$

H $-2.6616326294,2.43380102,1.3459832663$

O $0.7626163118,0.5743857355,-0.0487056056$

H $\quad 0.6584270603,1.6999150022,1.640190974$

C $0.3589002383,-0.6698924369,0.6650981783$

C $-1.1823086705,-0.6703300746,0.6782468094$

H $0.7667298086,-0.593345494,1.6802326229$

H $-1.5409857458,-0.7170432572,-0.3534732644$

H $-1.4842026818,-1.6007730125,1.1763350003$

C $0.9960543577,2.9279602584,-0.1987245659$

\footnotetext{
${ }^{5}$ See Ref. 19 complete citation on pg. S2
} 
H $\quad 0.9448400313,2.7705525323,-1.277251739$

H $\quad 0.2993292926,3.7104201712,0.1228110442$

H $\quad 2.0017959292,3.2865086087,0.075620676$

C $1.0350471339,-1.8307978908,-0.0471725027$

H $2.1183157062,-1.6955111852,-0.0579356002$

H $\quad 0.8030515063,-2.74779758,0.5003428584$

H $-1.6856887004,0.5073369283,2.4905968718$

$\mathrm{Cl} \quad 0.4910508785,-2.0388698616,-1.7450200312$

$\mathrm{E}+\mathrm{ZPE}=-808.911058$

2-chloromethyl-6-methyl-4-tetrahydropyranyl cation $\mathbf{4 4}$

b31yp/6-31g(d)

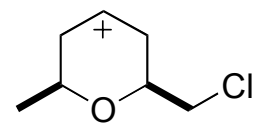

H $-1.5005751082,1.6807274366,-0.7142093649$

C $-1.5009694601,1.6800129841,0.3764159286$

C $0.175434849,1.6797692712,0.7345048591$

C $-1.8657005239,0.4552362304,0.998526918$

$\mathrm{H} \quad-1.9272471001,2.5852360442,0.8081422179$

O $0.6851372553,0.528565507,0.1660264543$

H $\quad 0.2669036035,1.681327686,1.8262511045$

C $0.2608085515,-0.6731393198,0.7218200615$

C $-1.3672223812,-0.7934953731,0.485592113$

H $\quad 0.4433929961,-0.7050671338,1.8040460106$

H $-1.4598898335,-0.9040383943,-0.5988851051$

H $\quad-1.7197964752,-1.6781730074,1.0186233705$

C $0.7842826558,2.9152443987,0.0989897974$

H $\quad 0.6880119811,2.8844458508,-0.9890853703$

H $\quad 0.3097343955,3.8209673455,0.4844462836$

H $1.8477298622,2.9474583865,0.3588411255$

C $0.9826379522,-1.8478994693,0.0658098761$

H $2.0548298576,-1.7311039691,0.2404767314$

H $\quad 0.6481401837,-2.7849841903,0.51473486$

H $-2.2865710799,0.4826586861,2.0049309838$

Cl $0.7258005185,-1.9413147723,-1.7044989297$

$\mathrm{E}+\mathrm{ZPE}=-808.905298$ 
chloromethyl cation 45 (open form 2)

b3lyp/6-31g(d)<smiles>C=CCC(C)OCCCl</smiles>

H $-1.3254089432,1.6811272418,-0.9557603014$

C $-1.328847766,1.7203434264,0.1381569063$

C $0.1369084683,1.756229176,0.6317076909$

C $-2.0809830977,0.5651525232,0.726945449$

H $-1.7788989285,2.6744230864,0.4380435704$

O $\quad 0.7036041484,0.4400641063,0.1956043705$

$\mathrm{H} \quad 0.1875095533,1.7599226047,1.7259238784$

C $0.4307720885,-0.604425514,0.8518666478$

C $-2.2821353699,-0.6027518388,0.0870665157$

H $\quad 0.0862351951,-0.5237511631,1.8847669038$

H $-1.9810146069,-0.7481969242,-0.9486595357$

H $-2.8270693423,-1.4178306706,0.55627991$

C $1.0007638247,2.8347188596,0.0162665534$

H $\quad 0.9891484064,2.7729060644,-1.0755898252$

H $\quad 0.6058781879,3.8114842619,0.3130063175$

H $2.0330640684,2.7646148493,0.369146187$

C $0.8272052328,-1.9444441705,0.345004248$

H $1.758346778,-2.2133177225,0.8692653875$

H $\quad 0.0746224438,-2.6798960131,0.6443155346$

H $-2.4444068536,0.681312621,1.7483863478$

$\mathrm{Cl} 1.1041800336,-2.0254262269,-1.4068260827$

$\mathrm{E}+\mathrm{ZPE}=-808.905118$

chloromethyl transition structure

(linking 43 and 44)

b3lyp/6-31g(d)

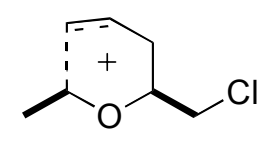

H $-1.6412367991,1.6366491204,-0.5404433306$

C $-1.6403490325,1.6366744121,0.5471224715$

C $0.310322435,1.6369732841,0.7538892337$

C $-1.7390997846,0.4360854673,1.2288828384$

$\mathrm{H} \quad-1.9899730128,2.5505833186,1.0215299841$

O $\quad 0.7048684157,0.5403697413,0.098994681$

$\mathrm{H} \quad 0.4306855749,1.6065514689,1.8393705424$

C $0.3553824549,-0.7108362267,0.6743866619$ 
C $-1.2367055246,-0.8136153354,0.6474711193$

H $\quad 0.7003112131,-0.7480275639,1.7159212771$

H $-1.5226738421,-0.9367024373,-0.4005351068$

H $-1.5221503804,-1.6959764402,1.2259506287$

C $0.7169879921,2.9241142213,0.0904019064$

H $\quad 0.5138209577,2.9047132649,-0.9822923918$

H $\quad 0.2250843503,3.7820071828,0.5536664349$

H $1.7994343841,3.0362641283,0.2383568589$

C $1.0411142201,-1.8306127181,-0.1006417382$

H $2.1226074579,-1.6873008132,-0.059868468$

H $\quad 0.7928873792,-2.7909374647,0.3560839292$

$\mathrm{H} \quad-1.976772564,0.4566323112,2.293010614$

Cl $0.5637124349,-1.8888301549,-1.8295166541$

Imaginary Frequency — -202.6151

$\mathrm{E}+\mathrm{ZPE}=-808.905143$

chloromethyl transition structure

(linking 44 and 45)

rb3lyp/6-31g(d)

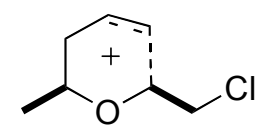

H $-1.3463824047,1.7639301074,-0.9480899655$

C $-1.3441506505,1.7639192871,0.1452172635$

C $0.1667920625,1.7681475174,0.6179794253$

C $-1.9755329503,0.555721017,0.7102899565$

H $-1.8027931103,2.6854741205,0.5143731666$

O $\quad 0.6938699633,0.4846665474,0.211762309$

H $\quad 0.2103503652,1.8280396257,1.7115361434$

C $0.2319220045,-0.5775174432,0.821685331$

C $-1.7812374614,-0.6890354424,0.1662431062$

H $\quad 0.0601091229,-0.5099499533,1.8986160804$

H $-1.4862292781,-0.8033126947,-0.8744620998$

H $-2.207021426,-1.5669653389,0.6465265786$

C $0.992652445,2.8572021607,-0.0375610456$

H $0.981430133,2.7535540152,-1.1261536525$

H $\quad 0.582328462,3.8358122658,0.2282210576$

H $\quad 2.0273142392,2.8135285167,0.3130355431$

C $0.7728840557,-1.9054951902,0.3584849292$

H $1.7809416077,-1.9893299046,0.7862527772$

H $\quad 0.1692085873,-2.7226695715,0.7568511501$

H $-2.439415192,0.6330545236,1.6940555773$

$\mathrm{Cl} \quad 0.9140718915,-2.0727146783,-1.4111114485$ 
Imaginary Frequency — - 185.7607

$\mathrm{E}+\mathrm{ZPE}=-808.902322$

vinyl substituted cation $\mathbf{4 9}$

b3lyp/6-31g(d)<smiles>C=CCC(C=C)OCC</smiles>

C $-0.1311446785,-1.347890392,-0.901502766$

O $-0.490575765,-0.7842454065,0.1668875214$

H $\quad 0.9087515069,-1.2562853599,-1.2229601957$

C $0.4305856811,0.229320433,0.8486468678$

H $1.4459292359,-0.147120837,0.690455385$

C $-1.065229679,-2.2215450185,-1.6308818816$

H $-2.0838261379,-2.1416610747,-1.2482927047$

H $-1.0263267908,-1.9929525735,-2.7025623101$

H $\quad-0.7089117605,-3.2589441336,-1.5289843988$

C $\quad 0.0597901091,0.2415480363,2.2898005432$

H $-0.9277612405,0.6285983436,2.5323936185$

C $0.9042514014,-0.1468577262,3.24687254$

H $1.8993927001,-0.5262301406,3.0248926166$

H $\quad 0.6334919944,-0.0851653516,4.2963385268$

C $0.1775037826,1.5448576921,0.0856990468$

H $\quad-0.8746466842,1.8246965691,0.2064674511$

H $\quad 0.7780057251,2.3065464594,0.5991008092$

C $0.5578052261,1.4551265103,-1.365208763$

H $1.6251092669,1.4534566302,-1.5894205647$

C $-0.324452738,1.3688213328,-2.3713844699$

H $-1.3980409614,1.4101276197,-2.1972308274$

H $-0.0012153632,1.3186118937,-3.4075442808$

$\mathrm{E}+\mathrm{ZPE}=-387.390441$

dienyl cation 51

b3lyp/6-31g(d)<smiles>C=CC=COC(C)CC=C</smiles>

H $\quad-2.2409633454,0.9759738935,-1.2081588298$

C $-2.3383658334,0.9203671291,-0.1257480854$

C $0.8788057012,0.768753279,0.2239975515$ 

C $-1.9683079721,-0.1686946141,0.560392348$
H $-2.7977848804,1.7737004891,0.3651489788$
O $\quad 0.8295230997,-0.3561883045,-0.3709291977$
H $\quad 0.5694197068,0.8312391676,1.2687966948$
C $0.1515227919,-1.5287061243,0.2715902588$
C $-1.3448603284,-1.3877711822,-0.0586868319$
H $\quad 0.3306094289,-1.4250493327,1.3476294384$
H $-1.465414662,-1.3873788329,-1.1476151652$
H $-1.8261853997,-2.2986772768,0.3209850151$
C $0.8213591891,-2.7647593108,-0.2924043605$
H $\quad 0.3767625949,-3.6498105017,0.1731361773$
H $0.6740384398,-2.8328822646,-1.374224608$
H $1.8931319865,-2.7691020387,-0.0763490424$
H $-2.1176383741,-0.1960947848,1.640601221$
C $1.4121981455,1.9080790281,-0.4484901136$
H $1.7051056032,1.7953904245,-1.4882907214$
C $1.5578081668,3.0706388272,0.2246939655$
H $1.2640128421,3.1741853611,1.2668696284$
H $1.9777620993,3.9505699402,-0.2531635993$

$\mathrm{E}+\mathrm{ZPE}=-387.403544$

vinyl transition structure 50 (linking 49 and 51 )

b3lyp/6-31g(d)

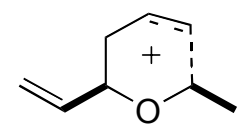

H $-1.7282261018,0.8083274459,-0.8599465226$

C $-1.3986412234,0.7854351039,0.1816497493$

C $0.2090235106,0.9296851327,0.1622082923$

C $-1.6756885665,-0.4895942195,0.8442643756$

$\mathrm{H}-1.7882836931,1.6507264542,0.7216318097$

O $0.6896751481,-0.2798925966,-0.4365900093$

H $0.573270281,1.0016412862,1.1926761357$

C $0.5105755287,-1.405655111,0.251931271$

C $-1.4498115117,-1.6970611557,0.2087707789$

H $0.6947499573,-1.3381844072,1.3265561343$

H - $1.531073841,-1.7566487522,-0.8740070451$

H $-1.6168241735,-2.6267996791,0.7473578774$

C $1.0396497212,-2.6376193683,-0.4285707508$

H $0.7064566663,-3.5454819553,0.0787483128$

H $0.7556429938,-2.668409605,-1.4825420942$

H 2.1352796267,-2.5981915539,-0.3645775947

H - $1.8158274528,-0.4870429406,1.9257108334$

C $0.6369518956,2.1014303497,-0.6574436195$

H $\quad 0.402359192,2.050110972,-1.7187637991$ 
C $1.2428948405,3.1641690666,-0.1278709608$

H $1.4809887691,3.2299456414,0.9314678294$

H $1.5243614213,4.0144090751,-0.7412266179$

Imaginary Frequency - -245.0639

$\mathrm{E}+\mathrm{ZPE}=-387.382672$

2,6-cis-Dimethyltetrahydropyran

b3lyp/6-31g(d)

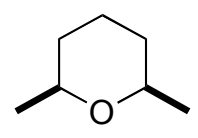

C $-1.2595398695,0.217072842,-1.1115956515$

C $-1.1981527674,0.1821054533,0.4212692925$

C $1.1976762673,0.1818322157,0.4227353032$

C $1.2609481468,0.2167841489,-1.1100533926$

C $0.0011342088,0.8750649027,-1.6905295041$

H $-1.1921681743,1.2238996652,0.7934789554$

H $-1.3439021145,-0.8153310725,-1.4786167231$

H $\quad-2.1618121481,0.7528014949,-1.4341800027$

H $1.1914737338,1.2236290175,0.7949349666$

H $2.1637368275,0.7523072597,-1.4315321493$

H $1.3455240141,-0.81563888444,-1.4769713334$

H $\quad 0.0011019578,1.9454366398,-1.4367635164$

H $\quad 0.0017970443,0.8134791051,-2.7856339142$

O $-0.0005777933,-0.4626879427,0.8613408705$

C $-2.3606783415,-0.569997372,1.0548880018$

H $-2.26381895,-0.5748986344,2.1451796248$

H $\quad-3.3151785107,-0.1013917326,0.7904134427$

H $\quad-2.3754601934,-1.6093423189,0.7083371234$

C $2.3592553566,-0.5705314743,1.0577784892$

H $3.3141850218,-0.1021460163,0.7944673179$

H $2.2610632631,-0.575402992,2.147951025$

H $2.3742225691,-1.6098822873,0.7112529876$

$\mathrm{E}+\mathrm{ZPE}=-350.206662$

cis-2-chloromethyl-6-methyltetrahydropyran<smiles>CC1CCCC(CCl)O1</smiles>

H $-1.4503851518,1.8329334662,-0.8345427564$

C $-1.4270447089,1.7427109976,0.2600589143$ 

C $0.0376464873,1.7232905876,0.7133264431$
C $-2.1476814954,0.4582794179,0.6931744444$
H $-1.928013497,2.6279439149,0.6724627283$
O $\quad 0.6972435936,0.544821565,0.2347210722$
$\mathrm{H} \quad 0.062031518,1.7090433682,1.8192305451$
C $0.102970716,-0.6663573354,0.6765733993$
C $-1.3595893268,-0.776154902,0.2307897278$
H $\quad 0.1321203128,-0.6970712222,1.785035079$
H $-1.3877076521,-0.8506714547,-0.8621492162$
H $-1.8009504931,-1.69626289,0.6355072884$
C $0.8450020691,2.9098030486,0.2064425004$
$\mathrm{H} \quad 0.8406391067,2.9325962249,-0.8886954348$
H $\quad 0.4213918477,3.8497804815,0.5770132712$
H $\quad 1.8839138782,2.837829597,0.543135921$
C $1.0046147428,-1.8097817012,0.2310753488$
H $2.0315503769,-1.6122669783,0.5416803869$
H $\quad 0.6637303939,-2.7537206721,0.6612969412$
H $\quad-2.2428662881,0.4449316064,1.7891474702$
$\mathrm{Cl} 1.0604856314,-2.0533232779,-1.569178005$
H $-3.1671697389,0.4321170845,0.2904906148$

$\mathrm{E}+\mathrm{ZPE}=-809.807461$

2,6-cis-dimethyl tetrahydropyranyl cation $\mathbf{4 7}$

b3lyp/6-31g(d)

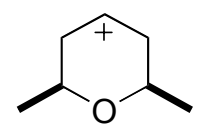

H $-1.2432218227,1.2293338356,-1.2386684875$

C $-1.2819966755,1.2018174261,-0.1486880547$

C $0.3521214718,1.1926895917,0.2971843501$

C $-1.7149088591,-0.0362863903,0.4167447221$

$\mathrm{H} \quad-1.7271503445,2.0999325941,0.2801851374$

O $\quad 0.8874784909,0.0188861959,-0.2105308447$

H $\quad 0.3821094777,1.2137313726,1.3923268424$

C $0.4140457379,-1.1587860584,0.3472590166$

C $-1.2168477688,-1.2728302115,-0.0960006991$

H $\quad 0.4438614801,-1.1316153364,1.4422710804$

H $-1.1754427689,-1.3446057977,-1.1838660401$

H $-1.6146135347,-2.1740647103,0.3711853163$

C $1.0249829478,2.4042556087,-0.3208372313$

H $0.9948165702,2.3553202531,-1.4122814406$

H $\quad 0.5423001295,3.3251251308,0.0160320549$

H $2.070613734,2.4260790874,0.0027896969$

C $1.150391894,-2.3584770133,-0.2194150425$

H $\quad 0.7164362756,-3.2882949061,0.1568631068$ 
H $1.1189195123,-2.3576700853,-1.3119184694$

H 2.1953592635,-2.311483931,0.1036791658

H $\quad-2.1705483881,-0.0271747925,1.4081664265$

$\mathrm{E}+\mathrm{ZPE}=-349.311968$

$\underline{\text { trimethyl cation } \mathbf{5 3}}$

b3lyp/6-31g(d)<smiles>C=C(C)CC(C)OCC</smiles>

C $1.3406863486,-0.5066549333,0.1538023121$

H $1.3397695199,-0.5440642002,1.2524228579$

H $2.3952958122,-0.4548692138,-0.1463809939$

C $0.7650526397,0.8452811592,-0.2825477683$

H $1.3364445551,1.6643897189,0.1677167666$

O $-0.6351489566,0.9778570793,0.3571266468$

C $-0.879866561,1.8106416199,1.2588093168$

H $-0.0696291052,2.4705017757,1.5938422767$

C $-2.21636477,1.9115683115,1.8572296279$

$\mathrm{H}-2.1275565114,1.771706692,2.945213163$

H $-2.5838905369,2.9406181958,1.7250239266$

H $-2.9173891356,1.1934718461,1.4298874437$

C $0.5483367016,1.0482866315,-1.7649559671$

H $1.524258319,0.9857373161,-2.2583872845$

H $\quad-0.0888121232,0.2663900396,-2.1840060727$

H $\quad 0.1228476223,2.032301349,-1.9808385844$

C $0.7019520973,-1.7528713488,-0.4322330093$

C $1.3238433587,-2.4301587624,-1.4033707145$

H $2.2845072089,-2.1175130499,-1.8051521204$

H $\quad 0.8993869962,-3.3372688289,-1.8240943342$

C $-0.610749603,-2.2095552245,0.158202049$

H $-0.5247676544,-2.3562523059,1.2435273626$

H $-1.4152585614,-1.4800835044,-0.0036735705$

H $-0.931356024,-3.1571471837,-0.2817290897$

$\mathrm{E}+\mathrm{ZPE}=-\mathbf{3 8 8 . 6 0 3 3 8 7}$ 
2,4,6-trimethyltetrahydropyranyl cation $\mathbf{5 4}$

b3lyp/6-31g(d)

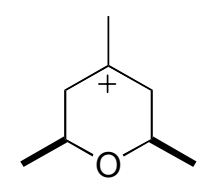

H $-1.1148621321,1.0993082032,-1.3948047756$

C $-1.0301612651,1.148957405,-0.3072915062$

C $0.5954218255,1.2222346501,-0.0321157442$

C $-1.4135860795,-0.0701280661,0.371963117$

H $-1.4859435581,2.0565475072,0.0927188413$

O $1.1497771903,0.0429669383,-0.5295162087$

H $\quad 0.7389656053,1.3006683921,1.0538477572$

C $0.7503707966,-1.128449658,0.1135797794$

C $-0.8686397557,-1.3027737696,-0.1553861867$

H $\quad 0.8940711669,-1.0533201389,1.1997562067$

H $\quad-0.9501975841,-1.3986607487,-1.2400403573$

H $-1.2050230095,-2.2060700207,0.3568273363$

C $-2.0820247862,-0.032552001,1.6893368204$

H $-1.8203299942,-0.8836015371,2.3257767563$

H $-1.9388424559,0.9170261127,2.2143314164$

H $\quad-3.1665844597,-0.1162032304,1.4912997575$

C $1.1822781454,2.42011036,-0.7585237672$

H $1.0443951165,2.3261798011,-1.8390953075$

H $\quad 0.7153452945,3.3466119065,-0.4136875053$

H $2.25406228,2.4741151743,-0.5447735594$

C $1.4946334258,-2.3194887596,-0.464737913$

H $1.1499178891,-3.2495909929,-0.004931653$

H $1.35455749,-2.3778847114,-1.5475285916$

H 2.5624969888,-2.2063221874,-0.2545142495

$\mathrm{E}+\mathrm{ZPE}=-388.618971$ 